\title{
Nuclear angiography in convalescent phase of myocardial infarction Serial study of left ventricular performance
}

\author{
J CHRISTINE RODGER, ROBERT RAILTON, KENNETH C YOUNG, \\ ANIL K LAKHANI, MASOOD A K GHOURI
}

From the Medical Unit and Department of Medical Physics, Monklands District General Hospital, Airdrie, Lanarkshire, and West of Scotland Health Boards, Department of Clinical Physics and Bio-Engineering, Glasgow

SUMMARY Electrocardiograph-gated blood pool scans (anteroposterior and left anterior oblique projections) were recorded in 30 patients seven to 10 days after myocardial infarction. Left ventricular ejection fractions (mean $0.26 \pm 0.10$ ) were lower on average than values previously obtained in 11 normal subjects (mean $0.52 \pm 0.06$ ) and correlated broadly with the clinical assessment of left ventricular performance. Ejection fractions were lower in anterior (mean $0.21 \pm 0.09$ ) than inferior (mean $0.32 \pm 0.08$ ) infarcts. Abnormal wall motion was detected in 11 of 15 anterior infarcts and in six of 13 inferior infarcts: mean ejection fractions associated with global asynergy, segmental asynergy, and normal wall motion were $0.15,0.26$, and 0.36 , respectively.

Twenty-four patients were reinvestigated two months later. Though there was some change in the clinical status of eight patients, wall motion and ejection fraction were unchanged (mean difference $-0.005 \pm 0.036)$.

Twelve patients were reinvestigated six months after infarction. The ejection fraction for the group was significantly lower than the values obtained at 10 days and two months, and four individual changes were significant when compared with the first study. Changes in wall motion were observed in one patient.

From this radionuclide study, we conclude that ejection fraction and wall motion do not improve after the early convalescent phase of myocardial infarction.

Nuclear angiography is now widely used for the assessment of left ventricular performance. ${ }^{1-3}$ This paper presents the results of a serial radionuclide study of left ventricular ejection fraction and wall motion in patients convalescing from myocardial infarction.

\section{Patients}

\section{STUDY 1}

Thirty patients aged 35 to 78 (mean 56 ) years were investigated seven to 10 days after acute myocardial infarction, that is just before discharge from hospital. All had raised cardiac enzymes: 27 had $Q$ wave and ST-T changes of acute myocardial infarction and three had ST-T changes only. On electroReceived for publication 10 August 1979 cardiographic evidence, the site of acute infarction was anterior in 15 patients, inferior in 13, anterior and inferior in one, and true posterior in one: two patients had a history and electrocardiographic evidence of previous myocardial infarction. All patients were in sinus rhythm and all but three were normotensive.

Left ventricular performance was assessed clinically and radiographically and the patients were grouped as follows. In 14 patients, left ventricular failure was judged to have been absent at all times (group 1). In six patients, signs of failure were present soon after infarction but had resolved by the time of study (group 2). In 10 patients, signs of failure persisted at the time of study despite a full anti-failure regimen (group 3). All patients in groups 2 and 3 were on diuretics and eight were 
also on digoxin. Three group 1 patients were on beta-adrenergic blocking agents.

\section{STUDY 2}

Twenty-four patients were reinvestigated approximately two months after myocardial infarction. On clinical and radiographic evidence, four patients had deteriorated and four had improved since study 1 ; the clinical status of the remaining 16 patients was judged to be unchanged.

\section{STUDY 3}

Twelve patients were investigated for a third time approximately six months after myocardial infarction. On clinical and radiographic evidence, two patients had deteriorated since study 2 ; the clinical status of the remaining 10 patients was judged to be unchanged.

\section{Methods}

The patients were investigated at rest. After the intravenous injection of $20 \mathrm{mCi}$ of $99^{\mathrm{m}}$-technetiumlabelled human serum albumin, patients lay under a gamma camera ${ }^{\star}$ positioned to achieve optimum separation of the left and right ventricles in the left anterior oblique projection (typically $30^{\circ}$ with $10^{\circ}$ caudal tilt). Acquisition and analysis were carried out on a micro-computer. $\dagger$ The heart rate was determined automatically and the electrocardiograph signal was used to trigger 16 sequential images of the cardiac cycle. Acquisition was suspended if intervals outside \pm 20 per cent of the initial RR interval estimate were detected. Each image was acquired in a $64 \times 64$ data matrix and acquisition was terminated automatically when a preset count density of 200 counts/pixel over the left ventricle had been achieved in the end-diastole * Ohio-Nuclear 410. † Ohio-Nuclear VIP 450, image. The 16 images were then stored on magnetic tape for later analysis. This procedure was repeated to acquire images in the anteroposterior view.

Images underwent nue-point smoothing, and were displayed in a continuous cinematic mode. For background estimation, a light pen was used to define an area adjacent to the apex of the left ventricle in the left anterior oblique view. The count density in this area during the cardiac cycle was displayed. If this was reduced at end-systole, a new area further removed from the left ventricle was selected. The average background count density in the selected area was then subtracted from each frame. Background corrected images were displayed cinematically and the end-diastolic outline was defined by automatically selecting up to 16 points with the light pen. The area of interest of the left ventricle was defined by joining these points. Total counts within this region were calculated for each image and the average timeactivity curve for the left ventricle during the cardiac cycle was displayed. Ejection fraction (EF) was calculated from the maximum and minimum counts in the region of the left ventricle using the following equation:

$$
\mathrm{EF}=\frac{\mathrm{C} \max -\mathrm{C}_{\min }}{\mathrm{C} \max }
$$

where, $\mathrm{C} \max =$ background corrected counts at end-diastole, and $\mathrm{C}$ min = background corrected counts at end-systole.

We have previously measured the precision of the method by assessment of the same data by two observers. The standard deviation for a single measurement of ejection fraction was found to be 0.024 , and for differences in pairs of measurements 0.035 . Therefore, the difference in two estimates required to achieve 95 per cent probability is 0.07

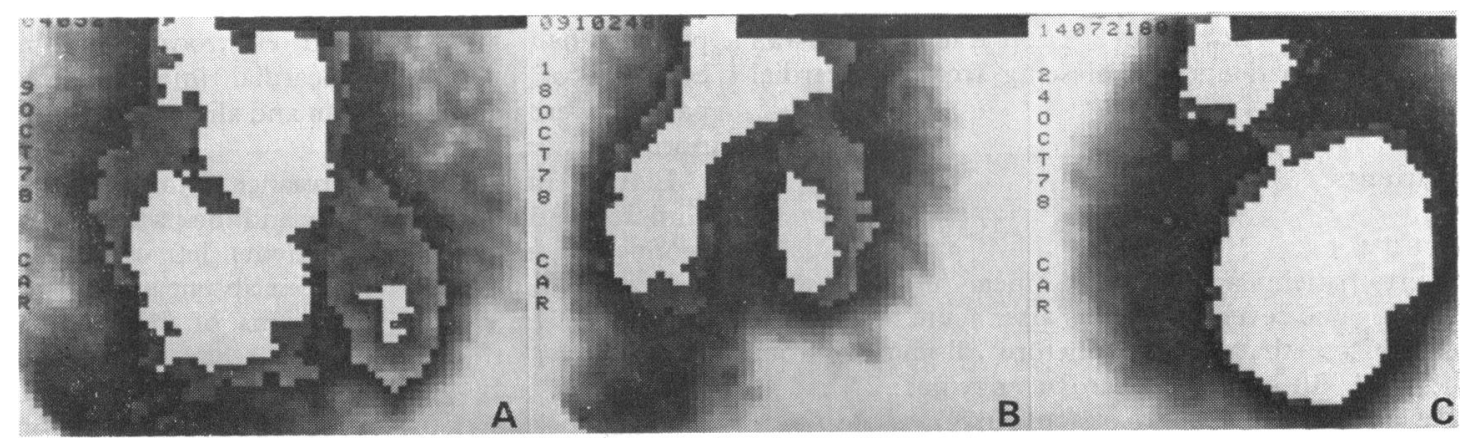

Fig. 1 Three categories of wall motion (see text) are illustrated: (A) no asynergy, (B) segmental asynergy, (C) global asynergy. LAO projection: end-systolic and end-diastolic images have been superimposed. 
and we have used this as our criterion for determining the significance of changes in ejection fraction.

The smoothed background corrected images were displayed cinematically and a line of equal count density (contour) was used to investigate the wall motion of the left ventricle in the anteroposterior and left anterior oblique views. This contour was adjusted by the operator until the left ventricular shape was visualised. The contour lines at end-diastole and end-systole were superimposed and recorded photographically. The relative wall motion was inferred from the degree of contour contraction in different directions from the centre (Fig. 1) and was classified as follows:

No asynergy: symmetrical contraction of the contours in all directions.

Segmental asynergy: reduced contraction of the contours in a particular direction.

Global asynergy: negligible contraction of the contours in all directions.

\section{Results}

STUDY 1

Left ventricular ejection fraction

Ejection fractions ranged from 0.09 to 0.45 (mean $0 \cdot 26 \pm 0 \cdot 10)^{\star}$ and were lower on average than values previously obtained in 11 normal subjects $(0.42$ to 0.62 : mean $0.52 \pm 0.06$ ).

Ejection fractions associated with anterior infarcts (mean $0.21 \pm 0.09)$ were significantly lower $(p<0.01)$ than ejection fractions associated with inferior infarcts (mean $0.32 \pm 0.08$ ).

In that the lowest ejection fractions were recorded in patients with persisting left ventricular failure (group 3) and the highest were recorded in patients without evidence of failure (group 1), the ejection fraction correlated broadly with the clinical assessment of left ventricular performance (Fig. 2). There was, however, some overlap between categories and only groups 1 and 3 differed significantly $(p<0.01)$.

Table Relation between wall motion and electrocardiographic site of infarction in study 1

\begin{tabular}{lllll}
\hline $\begin{array}{l}\text { Wall motion } \\
\text { category }\end{array}$ & \multicolumn{4}{l}{ Infarct site } \\
\cline { 2 - 5 } & Antelectroror & Inferior & $\begin{array}{l}\text { Anterior/ } \\
\text { inferior }\end{array}$ & $\begin{array}{l}\text { True } \\
\text { posterior }\end{array}$ \\
\hline No asynergy & 4 & 7 & 1 & 1 \\
Segmental asynergy & 4 & 4 & 0 & 0 \\
Global asynergy & 7 & 2 & 0 & 0 \\
\hline
\end{tabular}

\section{Wall motion}

In 13 patients there was no evidence of ventricular asynergy (Fig. 1A): segmental asynergy (Fig. 1B) $\star$ All error values quoted are standard deviations.

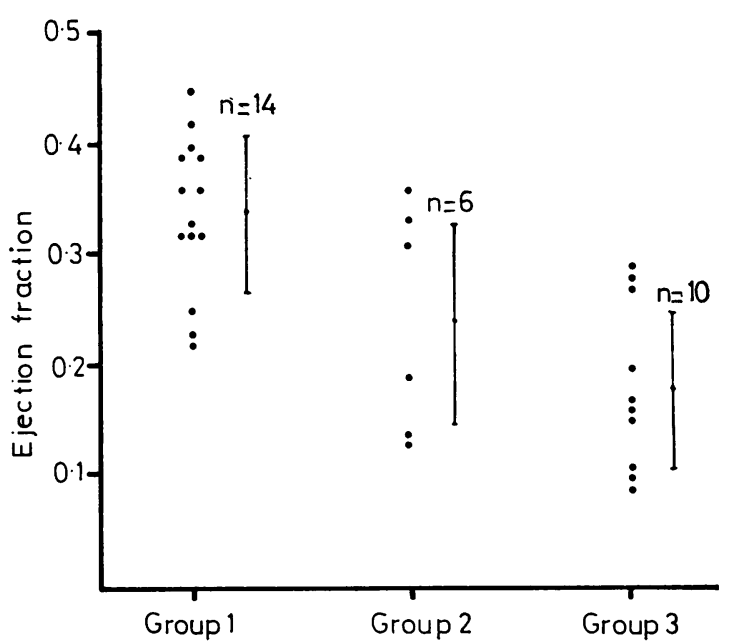

Fig. 2 Left ventricular ejection fraction in three clinical groups (see text). Mean values and standard deviations for groups 1, 2, and 3 are $0.34 \pm 0.07,0.24 \pm 0.09$, and $0 \cdot 18 \pm 0.07$, respectively.

was detected in eight patients and global asynergy (Fig. 1C) in nine. The Table shows the relation between wall motion and electrocardiographic site of infarction. In the eight patients with segmental asynergy, the electrocardiographic site of infarction correlated with the wall motion abnormality in six instances.

In that the lowest ejection fractions were recorded in patients with global asynergy and the highest ejection fractions were recorded in patients without asynergy, wall motion correlated broadly with ejection fraction (Fig. 3).

\section{STUDY 2}

\section{Left ventricular ejection fraction}

Ejection fractions ranged from 0.08 to 0.55 (mean $0 \cdot 28 \pm 0 \cdot 11$ ). Values for the group were not significantly different (paired $t$ test) from those obtained in study 1 (mean difference $=-0.005 \pm 0.036$ ) and only one significant individual change $(+0 \cdot 10)$ was observed.

In 15 patients, both ejection fraction and clinical status were unchanged. In eight patients ejection fraction was unaltered but clinical status was judged to have changed. In one patient ejection fraction changed significantly but the clinical status was unchanged.

\section{Wall motion}

No changes were observed.

STUDY 3

Left ventricular ejection fraction

Ejection fractions (Fig. 4) ranged from 0.15 to 0.40 


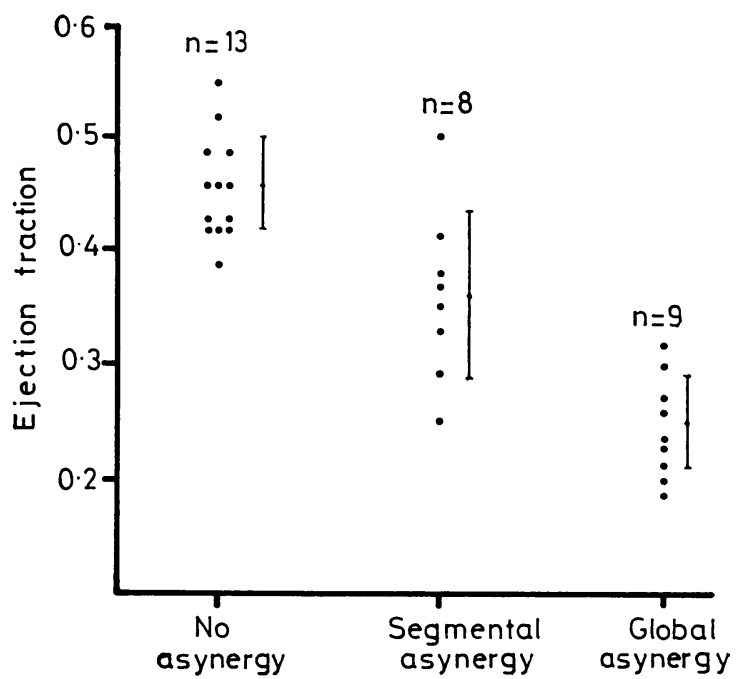

Fig. 3 Relation between left ventricular ejection fraction and wall motion. Mean values and standard deviations for no asynergy, segmental asynergy, and global asynergy are $0.36 \pm 0.04,0 \cdot 26 \pm 0 \cdot 07$, and $0 \cdot 15 \pm 0 \cdot 04$, respectively.

(mean $0 \cdot 27 \pm 0.07$ ). Values for the group were significantly $(\mathrm{p}<0.01$, paired $t$ test $)$ different from values obtained in study 2 (mean difference $=-0.031 \pm 0.026)$ but no individual change was significant. Thus, the clinical deterioration which had occurred in two patients was unaccompanied by a significant change in ejection fraction.

Values for the group were significantly $(p<0.001$, paired $t$ test) different from values obtained in study 1 (mean difference $=-0.045 \pm 0.030$ ) and the ejection fraction fell significantly in four patients. There was an accompanying change in clinical status in only one of these; patient $\mathrm{AH}$ (Fig. 4) had unstable angina at study 3.

\section{Wall motion}

Only one change was observed; patient AH (Fig. 4) had no evidence of ventricular asynergy at studies 1 and 2 but segmental asynergy was observed at study 3 (Fig. 5).

\section{Discussion}

There is ample evidence that the radionuclide ejection fraction can be measured reproducibly and that it is valid to use it in the serial assessment of left ventricular performance. ${ }^{3-5}$ Radionuclide ejection fractions have generally been found to be lower than values obtained by contrast angiography. ${ }^{34}$ The values obtained here in normal subjects agree well with those reported by others. ${ }^{6}$ 7
In the early convalescent phase (study 1) only two patients, one with electrocardiographic evidence of both fresh anterior and fresh inferior myocardial infarction, had ejection fractions within our normal range. The high incidence of abnormal ejection fractions in this phase and the broad correlation between ejection fraction and clinical assessment (Fig. 2) are in line with the findings of a previous nuclear angiographic study. ${ }^{8}$ The clinical status of most of our patients was unchanged over the months after infarction and in these instances the unaltered ejection fraction was in order. However, the discrepancies between ejection fraction and clinical assessment in studies 2 and 3 raise questions about the sensitivity of the radionuclide technique, the value of the resting ejection fraction as a measure of left ventricular performance, and the accuracy of our clinical judgment.

Ejection fraction as measured by nuclear angiography does not improve from the tenth day to the sixth month after myocardial infarction. Further, it appears on present evidence that ejection fraction tends to deteriorate over this period. There are obvious therapeutic implications which may merit further investigation. Thus, for example, the withdrawal of diuretic treatment in late convalescence from a patient shown to need it in the early convalescent phase seems to be inadvisable.

Schelbert et al. ${ }^{8}$ reported that the radionuclide ejection fraction improves between the early and late convalescent phases of myocardial infarction. Their baseline studies were performed within five days of infarction and thus possibly before their patients had achieved cardiovascular stability: this may explain the difference between their findings

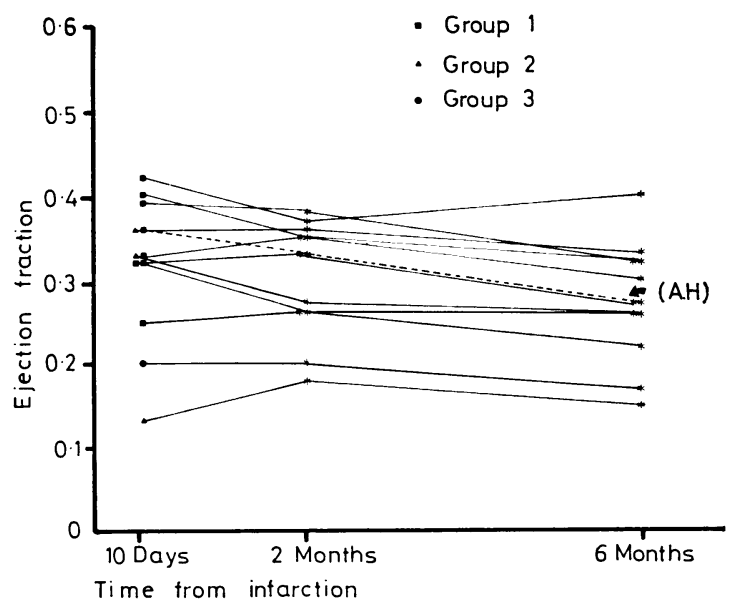

Fig. 4 Serial measurements of left ventricular ejection fraction in 12 patients. 


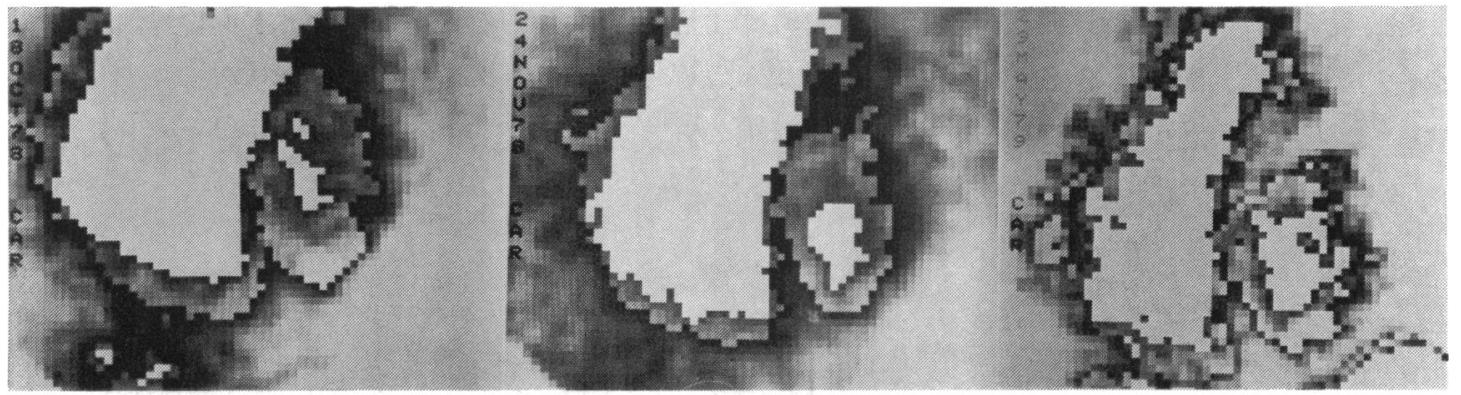

Fig. 5 Wall motion (LAO projection) in the same patient (AH) 10 days, two months, and six months after infarction: end-systolic and end-diastolic images are superimposed.

and ours. The present results are in line with the observations of Kupper et al. ${ }^{9}$ : they found almost no change in pulmonary artery end-diastolic pressure and cardiac index between the sixth week and tenth month after infarction.

Doubts have been expressed about the validity of radionuclide studies of wall motion. ${ }^{10}$ However, we were able to define three broad categories of wall motion, and believe that the isocount contour method can provide useful information on relative wall motion. The consistency of the method is illustrated in Fig. 6B, which was produced two months after Fig. 6A and without reference to it by an observer who had no knowledge of the infarct site or of the patient's clinical status.

The preponderance of wall motion abnormalities in patients with anterior infarcts (Table) could simply reflect inadequate imaging of the left ventricular inferior wall in the anteroposterior and left anterior oblique projections. However, the fact that the ejection fraction was lower in anterior than in inferior infarcts suggests that the difference in the incidence of wall motion abnormalities is real.

The correlation between left ventricular ejection fraction and wall motion (Fig. 3) is to be expected and has been reported previously. ${ }^{811}$ The patient in whom a change in wall motion was observed (Fig. 5) was one of four patients in whom ejection fraction fell significantly between the tenth day and the sixth month after infarction (Fig. 4).

Nuclear angiography provided information which was of some value in the practical management of this particular group of patients. Thus, one myocardial aneurysm was detected (Fig. 7), the

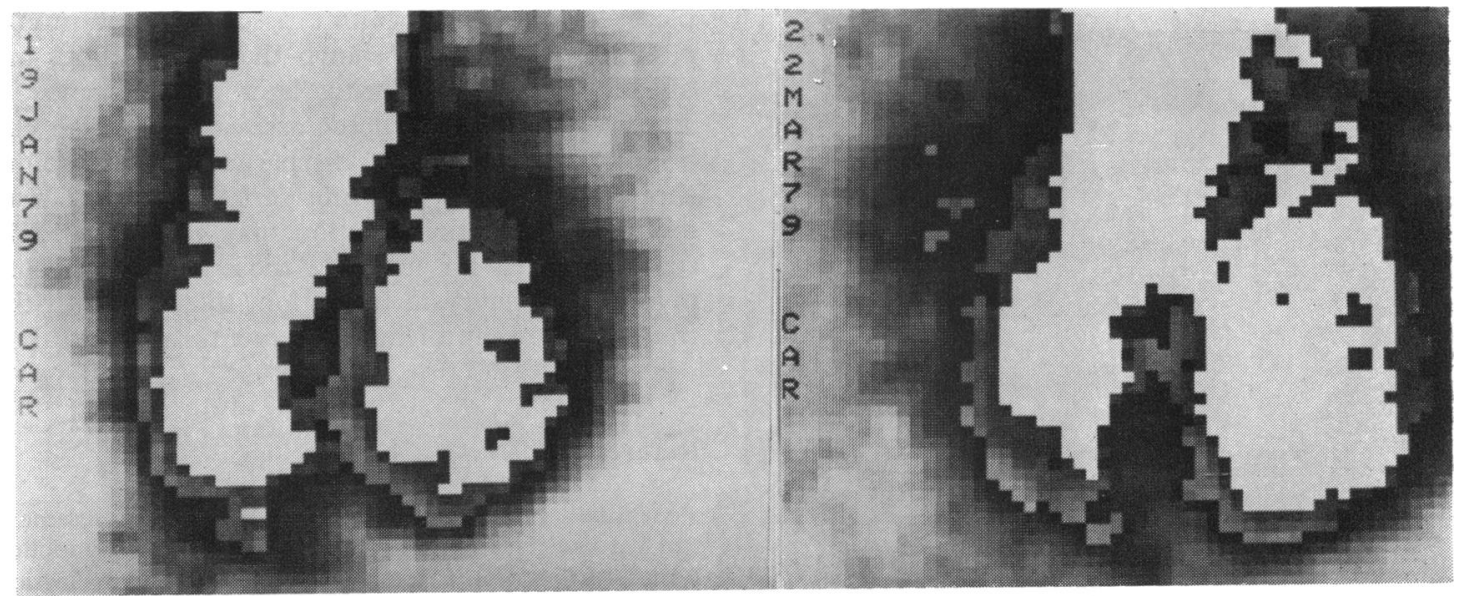

A

B

Fig. 6 Wall motion (LAO projection) in the same patient with an interval of two months between studies: end-systolic and end-diastolic images are superimposed. 


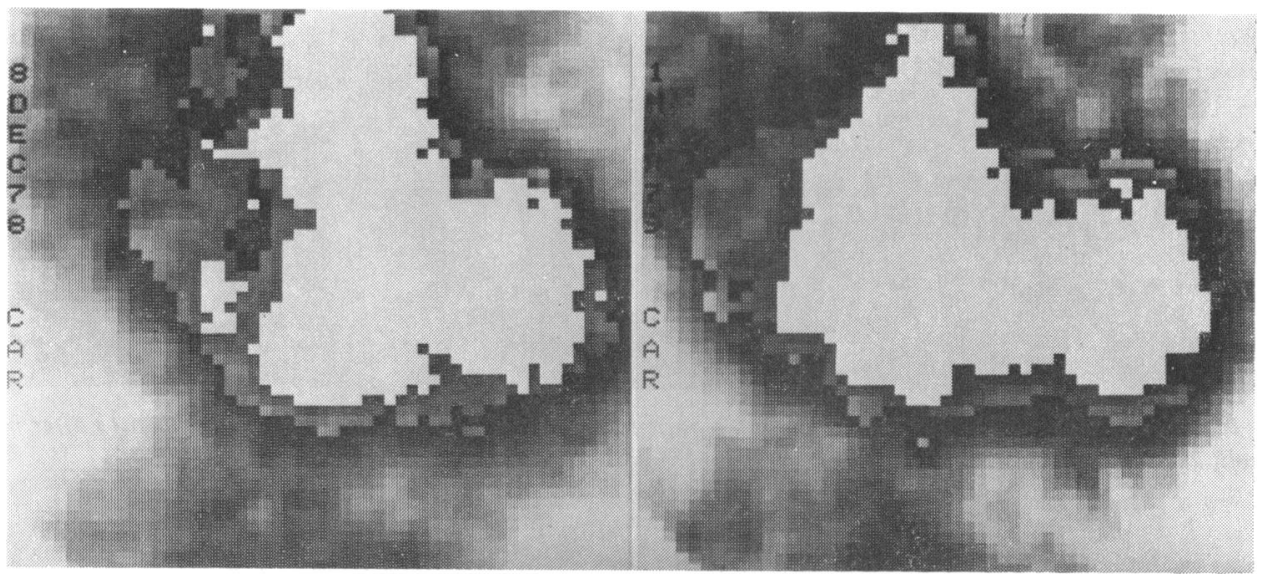

Fig. 7 Wall motion (AP projection) with an interval of three months between studies. Appearances suggest the development of an apical aneurysm.

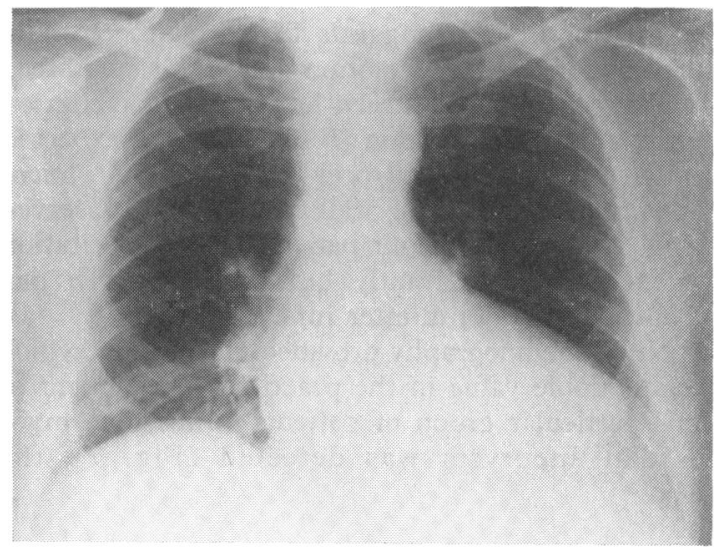

A

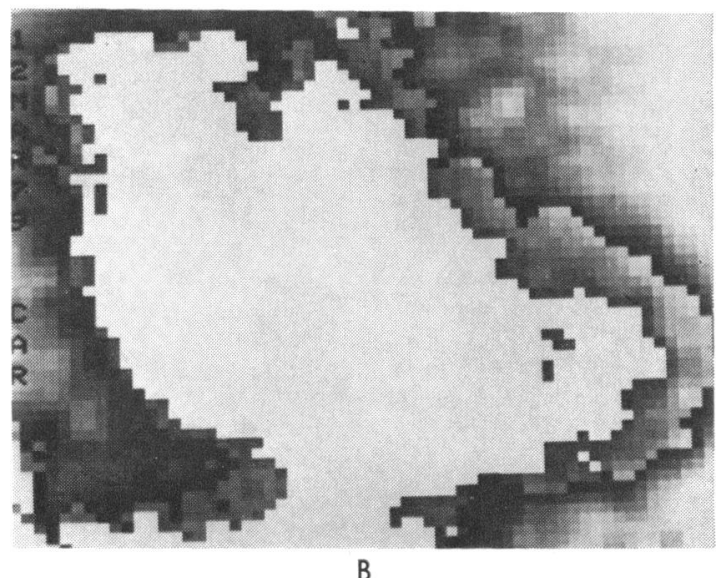

B

Fig. 8 (A) Chest $\mathrm{x}$-ray. (B) Wall motion in AP projection from a patient with possible right ventricular infarction (see text). possiblity of excising an infarcted segment was ruled out in the patients with global asynergy, and one patient with possible right ventricular infarction was identified (Fig. 8). This patient had an inferior infarct, a raised jugular venous pressure, cardiomegaly, and resistant supraventricular and ventricular arrhythmias. Despite these features, left ventricular ejection fraction was above average for the group, and there was no evidence of ventricular asynergy (Fig. 8B). The radionuclide images indicated that the radiographic appearances (Fig $8 \mathrm{~A}$ ) could be accounted for by right ventricular enlargement.

Nuclear angiography defines the extent to which left ventricular performance has been impaired by a myocardial infarction, and there seems to be a place for it in the routine assessment of infarct patients. As we have shown that the findings are unlikely to change during later convalescence, a single nuclear angiographic assessment, which can be undertaken before discharge from hospital, should be adequate. While serial studies of wall motion and ejection fraction may be indicated in some patients, for example those with suspected myocardial aneurysm formation, their value in the routine follow-up of infarct patients is doubtful.

\section{References}

${ }^{1}$ Marshall RC, Berger HJ, Costin JC, et al. Assessment of cardiac performance with quantitative radionuclide angiocardiography: sequential left ventricular ejection fraction, normalized left ventricular ejection rate and regional wall motion. Circulation 1977; 56: 820-9.

${ }^{2}$ Hecht HS, Mirell SG, Rolett EL, Blahd WH. Left ventricular ejection fraction and segmental wall 
motion by peripheral first-pass radionuclide angiography. .7 Nucl Med 1978; 19: 17-23.

${ }^{3}$ Ashburn WL, Schelbert HR, Verba JW. Left ventricular ejection fraction. A review of several radionuclide angiographic approaches using the scintillation camera. Prog Cardiovasc Dis 1978; 20: 267-84.

${ }^{4}$ Folland ED, Hamilton GW, Larson SM, Kennedy JW, Williams DL, Ritchie JL. The radionuclide ejection fraction; a comparison of three radionuclide techniques with contrast angiography. $f \mathrm{Nucl} \mathrm{Med}$ 1977; 18: 1159-66.

${ }^{5}$ Marshall RC, Berger HJ, Reduto LA, Gottschalk A, Zaret BL. Variability in sequential measures of left ventricular performance assessed with radionuclide angiocardiography. Am $\mathcal{f}$ Cardiol 1978; 41: 531-6.

${ }^{6}$ Borer JS, Bacharach SL, Green MV, Kent KM, Epstein MD, Johnston GS. Real-time radionuclide cineangiography in the noninvasive evaluation of global and regional left ventricular function at rest and during exercise in patients with coronary-artery disease. $N$ Engl f Med 1977; 296: 839-46.

'Qureshi S, Wagner HN Jr, Alderson PO, et al. Evaluation of left ventricular function in normal persons and patients with heart disease. $\mathrm{f}$ Nucl Med 1978; 19: 135-41.
${ }^{8}$ Schelbert HR, Henning H, Ashburn WL, Verba JW, Karliner JS, O'Rourke RA. Serial measurements of left ventricular ejection fraction by radionuclide angiography early and late after myocardial infarction. Am f Cardiol 1976; 38: 407-15.

${ }^{\circ}$ Kupper W, Bleifeld W, Hanrath P, Mathey D, Effert $S$. Left ventricular haemodynamics and function in acute myocardial infarction: studies during the acute phase, convalescence and late recovery. $\mathrm{Am} \mathcal{f}$ Cardiol $1977 ; 40$ : 900-5.

${ }^{10}$ Walton S, Rowlands DJ, Shields RA, Testa HJ, Wrigley C. Limitations of gated blood pool scintigraphy: clinical and experimental aspects (abstract). Br Heart f 1979; 41 : 373.

${ }^{11}$ Dymond DS, Stone DL, Elliot AT, Britton KE, Spurrell RAJ. Biplane first pass radionuclide ventriculography in assessment of wall motion abnormalities in patients with acute myocardial infarction (abstract). Br Heart f 1979; 41 : 378.

Requests for reprints to Dr J C Rodger, Medical Unit, Monklands District General Hospital, Airdrie, Lanarkshire ML6 0JS. 